\title{
Non-dipping nocturnal blood pressure correlates with obstructive sleep apnoea severity in normotensive subjects and may reverse with therapy
}

\author{
Sophie J. Crinion (i) ${ }^{1,2}$, Jana Kleinerova ${ }^{3}$, Brian Kent ${ }^{4}$, Geraldine Nolan ${ }^{5}$, Cormac T. Taylor ${ }^{6}$, \\ Silke Ryan (1D) ${ }^{5,6,7}$ and Walter T. McNicholas $\mathbb{1}^{5,6,7}$
}

${ }^{1}$ Respirology and Sleep Medicine, Kingston Health Sciences Centre, Kingston, Canada. ${ }^{2}$ Dept of Medicine, Queen's University, Kingston, Canada. ${ }^{3}$ Dept of Respiratory Medicine, Mater Misericordiae University Hospital, Dublin, Ireland. ${ }^{4}$ Dept of Respiratory Medicine, St James' Hospital, Dublin, Ireland. ${ }^{5}$ Dept of Respiratory and Sleep Medicine, St Vincent's University Hospital, Dublin, Ireland. ${ }^{6}$ School of Medicine, University College Dublin, Dublin, Ireland. ${ }^{7}$ These authors contributed equally.

Corresponding author: Walter T. McNicholas (walter.mcnicholas@ucd.ie)

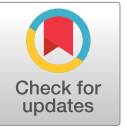

Copyright $\odot$ The authors 2021

This version is distributed under the terms of the Creative Commons Attribution NonCommercial Licence 4.0. For commercial reproduction rights and permissions contact permissions@ersnet.org

Received: 18 May 2021 Accepted: 9 June 2021

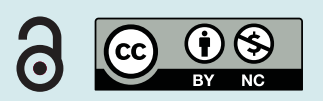

\begin{abstract}
Shareable abstract (@ERSpublications)
There is a strong association in a normotensive cohort between OSA and nocturnal blood pressure, with intermittent hypoxia as an important mechanistic pathway https://bit.ly/2SFvWiB
\end{abstract}

Cite this article as: Crinion SJ, Kleinerova J, Kent B, et al. Non-dipping nocturnal blood pressure correlates with obstructive sleep apnoea severity in normotensive subjects and may reverse with therapy. ERJ Open Res 2021; 7: 00338-2021 [DOI: 10.1183/23120541.00338-2021].

\section{Abstract}

Background Obstructive sleep apnoea (OSA) is strongly associated with systemic hypertension, but there are limited data on the relationship with blood pressure (BP) in normotensive subjects. Here, we examined the relationship of OSA with nocturnal BP in a documented diurnal normotensive cohort, explored potential intermediate pathways and assessed the effects on BP of continuous positive airways pressure (CPAP) therapy.

Methods 65 males referred for assessment of possible OSA and normotensive on 24-hour BP monitoring underwent overnight inpatient polysomnography (age $41 \pm 7$ years, body mass index (BMI) $34 \pm 6 \mathrm{~kg} \cdot \mathrm{m}^{-2}$, apnoea-hypopnoea index (AHI) 14 (interquartile range 5-26)). Urine and serum were assessed for markers of sympathetic activation, renin-angiotensin-aldosterone system activity, oxidative stress, endothelial function and systemic inflammation. In a subset of patients, 24-hour BP monitoring was repeated after CPAP therapy. Results Within this normotensive cohort, night-time systolic and diastolic BP and nocturnal BP dip were highest in the fourth OSA severity quartile $(\mathrm{p}<0.05)$. Nocturnal BP dip correlated with AHI $(\mathrm{r}=-0.327$, $\mathrm{p}<0.05)$ and oxygen desaturation index (ODI) ( $\mathrm{r}=-0.371, \mathrm{p}<0.05)$, but only ODI was an independent predictor of $\mathrm{BP} \operatorname{dip}(\mathrm{B}=-0.351, \mathrm{p}<0.01)$ and non-dipping status $(\mathrm{B}=0.046, \mathrm{p}<0.05)$. Overnight urinary norepinephrine correlated with nocturnal systolic BP $(r=0.387, p<0.01)$ with a trend towards correlation with systolic dipping $(\mathrm{p}=0.087)$. In 20 CPAP-treated patients, night-time systolic BP decreased $(\mathrm{p}<0.05)$ and mean nocturnal BP dip increased $(\mathrm{p} \leqslant 0.05)$.

Conclusion In this normotensive cohort, OSA severity was associated with higher nocturnal BP, which improved following CPAP therapy, and intermittent hypoxia was the most important OSA-related variable in this relationship.

\section{Introduction}

Obstructive sleep apnoea (OSA) is highly prevalent in the general population with an estimated global prevalence of close to one billion affected [1]. Hypertension is present in up to 50\% of patients with OSA, which is about double the prevalence of hypertension in general population studies [2]. A non-dipping nocturnal blood pressure (BP) profile ( $<10 \%$ day-to-night systolic blood pressure difference) is especially likely in OSA patients [3-6], even in the absence of significant hypertension. The likelihood of non-dipping BP correlates with OSA severity in population studies [7], and cardiovascular events are more frequent in OSA patients with a non-dipping BP profile than those with a normal dipping pattern, even in the absence of diagnosed hypertension [8]. Despite the extensive publications on the relationships between 
OSA and hypertension and responses to therapy, there are few data on the relationship between OSA and 24-hour BP patterns in OSA patients who are normotensive on 24-hour ambulatory BP monitoring (ABPM) and free of any cardiovascular disease or other chronic disorders. SAPIÑA-BELTRÁn and co-workers evaluated the impact of continuous positive airways pressure (CPAP) therapy on 24-hour BP levels in OSA patients who were normotensive on office BP measurements and demonstrated a significant difference in mean nocturnal BP between dippers and non-dippers after 6 months of CPAP therapy, the former showing an increase and the latter a decrease [9]. The potential for BP reduction or return of a dipping pattern in normotensive patients with OSA would provide an additional consideration in treatment decisions for individual patients and may also have implications for the possible primary prevention of hypertension in such patients. The question of primary prevention assumes additional significance in the context of the recent SAVE trial, which reported that CPAP therapy conferred no benefit in the secondary prevention of cardiovascular morbidity and mortality in patients with pre-existing cardiovascular disease [10].

Based on these considerations, we studied the relationship between nocturnal BP pattern and OSA in a prospective cohort of patients referred for investigation of suspected OSA who were proven normotensive on 24-hour ABPM and free of any cardiovascular or other comorbidity. The primary objective of the study was to examine the relationship of OSA with nocturnal BP dipping and other BP variables in such normotensive subjects. Secondary objectives were: (1) to explore a range of potential intermediate pathways in this relationship that have previously been associated with OSA, hypertension and other cardiovascular diseases; and (2) to assess the potential effects of therapy on nocturnal BP pattern in patients from the cohort who were diagnosed with OSA and commenced on CPAP therapy.

\section{Methods}

\section{Subjects}

Consecutive patients referred to the Sleep Clinic in St Vincent's University Hospital were screened for inclusion to the study and were considered if they were male and aged 18 to 70 years without a known diagnosis of hypertension. Exclusion criteria included any previous investigation or diagnosis of OSA, history of cardiovascular disease (including hypertension, coronary artery disease or previous myocardial infarction), metabolic disease (including diabetes), thyroid disease or any requirement for long-term medication. Consenting participants were included if normotension was confirmed on ABPM. Prior approval was obtained from the Ethics and Medical Research Committee of St Vincent's Healthcare Group, Dublin, Ireland, and all subjects provided written informed consent.

\section{Study protocol}

Polysomnography

Subjects underwent an attended, inpatient polysomnography (PSG) in the Sleep Laboratory at St Vincent's University Hospital, Dublin, Ireland. Following the AASM 2012 [11] Scoring Manual, a $\geqslant 90 \%$ decrease in oronasal airflow below pre-event baseline for $\geqslant 10 \mathrm{~s}$ during sleep resulted in an apnoea, and a $\geqslant 30 \%$ decrease in oronasal airflow below pre-event baseline accompanied by either a $\geqslant 3 \%$ decrease in oxygen saturations or an arousal from sleep resulted in the scoring of a hypopnoea.

\section{ABPM}

On the morning after PSG, an ABPM was fitted to the subject's non-dominant arm (WatchBP03; Microlife Corp., Taipei, Taiwan). The patient's self-reported routine sleep and wake times were programmed into the device and this information was rechecked with the patient when it was returned. BP measurements were taken every 20 minutes during daytime (awake) hours and 30 minutes during night-time (asleep) hours to ensure at least one measurement per hour.

\section{Biomarkers of intermediate pathways}

Patients were instructed to void their bladder before getting into bed for the PSG. All urine voided throughout the night including the first void on waking in the morning was collected in a polyethylene container; $100 \mathrm{~mL} 1 \mathrm{M}$ hydrochloric acid was added to the container prior to the collection as a preservative agent. Samples were analysed immediately after collection in the laboratory of St Vincent's University Hospital using high-performance liquid chromatography to determine urinary noradrenaline $\left(\mathrm{nmol} \cdot \mathrm{L}^{-1}\right)$ for measurement of urinary catecholamines.

In the morning following the PSG, venous blood samples were taken from each patient in a fasting state using BD Vacutainers (Becton, Dickinson and Company, Franklin Lakes, USA). Two samples with added silica and one sample with added lithium heparin were centrifuged for 15 minutes at $1800 \mathrm{xg}$ and $4^{\circ} \mathrm{C}$ to separate plasma and serum, respectively. Following centrifugation, the samples were separated into $0.5-\mathrm{mL}$ aliquots in sterile cryogenic vials and stored at $-80^{\circ} \mathrm{C}$ for future analysis. After collection of all patient 
samples, analysis was done using commercially available sandwich enzyme-linked immunosorbent assays (ELISA) for the detection of plasma renin, interleukin-6 (IL-6), tumour necrosis factor- $\alpha$ (TNF- $\alpha$ ), oxidised low-density lipoprotein (oxLDL) and endothelin 1.

CPAP

Subjects with OSA diagnosed on PSG were subsequently offered therapy with CPAP as per the departmental protocol, including attended inpatient CPAP initiation and titration. Subjects who accepted CPAP therapy were contacted and invited to return for repeat ABPM testing as per the protocol described above.

\section{Statistical analysis}

Data analysis was done using IBM SPSS Statistics for Windows (Version 20, Armonk, NY, USA). Patient characteristics and ABPM results were categorised into OSA severity quartiles based on the AHI. Data are displayed as mean (SD) or median (interquartile range) depending on distribution. Groups were compared by one-way ANOVA followed by Bonferroni post hoc comparison. Patient characteristics were univariately correlated with \% BP dip and non-dipping status. Linear and logistic regression models were built to calculate predictors of \% BP dip and non-dipping status. Paired t-test and McNemar's test were used to compare measured variables and ratio of non-dippers respectively in subjects' pre- and post-CPAP therapy.

\section{Results}

\section{Baseline characteristics}

65 consecutive male patients were included in the study. Baseline characteristics of the study population, stratified by AHI quartiles, are described in table 1. The four groups were similar with regard to age, smoking status, lipid levels, renal function and Epworth sleepiness score. However, subjects with more severe OSA were more obese, had higher levels of glycosylated haemoglobin (HbA1c) and increased insulin resistance as calculated by HOMA-IR.

\section{TABLE 1 Total cohort patient characteristics and blood pressure measurements stratified by obstructive sleep apnoea (OSA) severity quartiles}

\begin{tabular}{|c|c|c|c|c|c|c|}
\hline Variable & Total cohort & Quartile 1 & Quartile 2 & Quartile 3 & Quartile 4 & $\mathrm{p}$-value \\
\hline Subjects $n$ & 65 & 16 & 17 & 16 & 16 & \\
\hline Age years & $41 \pm 7$ & $38 \pm 6$ & $40 \pm 8$ & $42 \pm 8$ & $43 \pm 7$ & 0.158 \\
\hline BMI $\mathrm{kg} \cdot \mathrm{m}^{-2}$ & $33.5 \pm 5.8$ & $30.1 \pm 3.7$ & $30.9 \pm 2.6$ & $34.8 \pm 5.0^{\#}$ & $38.5 \pm 6.9^{\#, \bullet}$ & $<0.001$ \\
\hline Neck circumference $\mathrm{cm}$ & $43 \pm 3$ & $41 \pm 3$ & $43 \pm 2$ & $44 \pm 3^{\#}$ & $45 \pm 3^{\#}$ & 0.004 \\
\hline Waist-hip ratio & $1.00 \pm 0.06$ & $0.98 \pm 0.07$ & $1.00 \pm 0.06$ & $1.00 \pm 0.04$ & $1.04 \pm 0.05$ & 0.115 \\
\hline Smoking pack-years & $9 \pm 12$ & $9 \pm 7$ & $8 \pm 13$ & $13 \pm 14$ & $7 \pm 10$ & 0.566 \\
\hline Current smokers \% & 20 & 25 & 18 & 25 & 13 & 0.862 \\
\hline Clinic systolic BP & $137 \pm 11$ & $136 \pm 14$ & $136 \pm 11$ & $140 \pm 12$ & $138 \pm 8$ & 0.706 \\
\hline Clinic diastolic BP & $83 \pm 8$ & $83 \pm 9$ & $81 \pm 7$ & $84 \pm 9$ & $86 \pm 7$ & 0.458 \\
\hline ESS score & $11 \pm 5$ & $10 \pm 7$ & $12 \pm 5$ & $12 \pm 5$ & $12 \pm 5$ & 0.885 \\
\hline HBA1c & $37 \pm 3$ & $36 \pm 2$ & $35 \pm 3$ & $36 \pm 3$ & $39 \pm 3^{\#, \uparrow,+}$ & 0.004 \\
\hline HOMA & $3.7 \pm 2.9$ & $2.2 \pm 1.1$ & $3.3 \pm 1.9$ & $3.2 \pm 1.9$ & $6.0 \pm 4.1^{\#, \triangleleft,+}$ & 0.001 \\
\hline Total cholesterol & $4.9 \pm 0.8$ & $5.1 \pm 0.8$ & $4.8 \pm 0.7$ & $5.0 \pm 0.9$ & $4.8 \pm 1.0$ & 0.652 \\
\hline Triglycerides & $1.6 \pm 0.8$ & $1.5 \pm 0.9$ & $1.5 \pm 0.7$ & $1.9 \pm 1.0$ & $1.5 \pm 0.8$ & 0.362 \\
\hline $\mathrm{HDL}$ & $1.1 \pm 0.3$ & $1.1 \pm 0.3$ & $1.2 \pm 0.3$ & $1.1 \pm 0.3$ & $1.1 \pm 0.3$ & 0.851 \\
\hline LDL & $3.1 \pm 0.7$ & $3.3 \pm 0.6$ & $3.0 \pm 0.7$ & $3.1 \pm 0.7$ & $3.0 \pm 0.9$ & 0.528 \\
\hline Creatinine & $84 \pm 12$ & $89 \pm 14$ & $85 \pm 14$ & $84 \pm 11$ & $79 \pm 8$ & 0.110 \\
\hline eGFR & $97 \pm 13$ & $95 \pm 15$ & $97 \pm 14$ & $96 \pm 10$ & $102 \pm 11$ & 0.400 \\
\hline $\mathrm{AHI}$ events $\cdot \mathrm{h}^{-1}$ & $13.6(4.7-26.2)$ & $1.3(0.6-3.4)$ & $7.7(5.6-14.7)$ & $19.7(17.0-24.6)^{\#}$ & $45.0(33.5-75.6)^{\#, \bullet,++}$ & $<0.001$ \\
\hline ODI events $\cdot \mathrm{h}^{-1}$ & $12.2(3.9-25.1)$ & $1.8(0.6-2.3)$ & $6.4(5.5-7.8)$ & $17.2(14.6-20.3)^{\#}$ & $44.9(26.6-62.5)^{\#, \bullet,++}$ & $<0.001$ \\
\hline TST90 \% & $0.6(0.0-5.6)$ & $0.0(0-0.2)$ & $0.1(0-1.3)$ & $2.2(0.2-4.9)$ & $19.8(3.8-54.6)^{\#, \bullet,++}$ & $<0.001$ \\
\hline Minimum $\mathrm{SpO}_{2} \%$ & $87(79-89)$ & $89(88-91)$ & $88(85-90)$ & $83(78-87)^{\#}$ & $72(67-84)^{\#, 9,+}$ & $<0.001$ \\
\hline Arousal index events $\cdot \mathrm{h}^{-1}$ & $8.3(5.2-16.4)$ & $5.6(3.8-7.1)$ & $9.0(5.6-14.7)$ & $9.1(4.5-12.7)$ & $18.9(7.0-31.7)^{\#, \boldsymbol{\bullet},+}$ & 0.001 \\
\hline
\end{tabular}


TABLE 2 Total cohort blood pressure measurements stratified by obstructive sleep apnoea (OSA) severity quartiles

\begin{tabular}{lcccccc} 
Variable & Total cohort & Quartile 1 & Quartile 2 & Quartile 3 & Quartile 4 & p-value \\
\hline ABPM day systolic & $121 \pm 9$ & $120 \pm 9$ & $121 \pm 9$ & $120 \pm 9$ & $122 \pm 7$ & 0.851 \\
\hline ABPM day diastolic & $74 \pm 9$ & $73 \pm 8$ & $74 \pm 7$ & $71 \pm 13$ & $77 \pm 7$ & 0.355 \\
\hline ABPM night systolic & $112 \pm 10$ & $107 \pm 10$ & $108 \pm 9$ & $113 \pm 9$ & $119 \pm 7^{\#, 9}$ & 0.001 \\
\hline ABPM night diastolic & $66 \pm 8$ & $61 \pm 9$ & $65 \pm 8$ & $69 \pm 9$ & $68 \pm 6$ & 0.039 \\
\hline ABPM \% dip & $7.3 \pm 7.7$ & $11.0 \pm 6.0$ & $9.3 \pm 7.2$ & $5.4 \pm 9.2$ & $3.2 \pm 5.9^{\#}$ & 0.012 \\
\hline \% non-dippers & 66 & 56 & 59 & 63 & 88 & 0.216 \\
\hline \% below daytime value of $135 / 85 \mathrm{mmHg}$ & 92 & 94 & 100 & 88 & 88 \\
\% below night-time value of $120 / 70 \mathrm{mmHg}$ & 69 & 75 & 77 & 69 & 56 \\
\hline
\end{tabular}

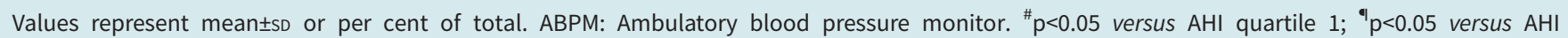
quartile 2 .

24-hour BP recordings

ABPM was obtained in all subjects. As shown in table 2, all subjects were normotensive and there was no difference in daytime values between AHI quartiles. However, nocturnal BP increased with higher AHI, and subjects in the 4th AHI quartile had a significantly lower dipping ratio in comparison to subjects in quartile 1. Univariate linear correlation analysis showed a significant inverse correlation between the percentage in BP dip and body mass index (BMI), neck circumference and markers indicating the severity of OSA. The presence of a non-dipping BP status correlated with increasing AHI, oxygen desaturation index (ODI) and lower minimal oxygen saturation values (table 3). Stepwise multiple linear regression analysis identified the ODI and the amount of smoking (pack-years) as independent predictors of BP dip (table 4). Furthermore, subjects in AHI quartile 4 versus quartile 1 had increased odds of a non-dipping $\mathrm{BP}$ status, but this failed to reach statistical significance $(\mathrm{p}=0.063)$ (data not shown). However, increasing severity of ODI, but not AHI, conferred significantly increased odds of the non-dipping status, independent of age, BMI, smoking status and insulin sensitivity (table 4).

Relationship between BP dip and biomarkers of potential intermediate pathways

To gain insight into potential mechanistic pathways underlying the association of OSA and non-dipping $\mathrm{BP}$, we performed a correlation analysis of BP values with markers of sympathetic activation, renin-

TABLE 3 Univariate correlation of \% blood pressure dip and non-dipping status with patient parameters

\begin{tabular}{|c|c|c|c|c|c|}
\hline \multirow[b]{2}{*}{ Variable } & \multicolumn{2}{|c|}{$\%$ Blood pressure dip } & \multicolumn{3}{|c|}{ Non-dipping status } \\
\hline & Correlation coefficient & p-value & Unstandardised $\boldsymbol{\beta}(95 \% \mathrm{CI})$ & Standardised $\boldsymbol{\beta}$ & p-value \\
\hline Age & -0.067 & 0.594 & $0.989(0.923-1.060)$ & -0.11 & 0.759 \\
\hline BMI & -0.248 & 0.022 & $1.096(0.987-1.218)$ & 0.092 & 0.086 \\
\hline Neck circumference & -0.373 & 0.004 & $1.147(0.952-1.383)$ & 0.138 & 0.149 \\
\hline Waist-hip ratio & -0.168 & 0.258 & $1.223(0.00-50465.75)$ & 0.202 & 0.970 \\
\hline Smoking pack-years & -0.238 & 0.061 & $0.975(0.928-1.025)$ & -0.025 & 0.318 \\
\hline HbA1c & -0.192 & 0.125 & $1.139(0.963-1.349)$ & 0.131 & 0.129 \\
\hline HOMA & -0.227 & 0.087 & $1.170(0.909-1.507)$ & 0.157 & 0.223 \\
\hline Creatinine & -0.007 & 0.955 & $1.00(0.959-1.043)$ & 0.000 & 0.990 \\
\hline eGFR & 0.047 & 0.710 & $1.001(0.961-1.044)$ & 0.001 & 0.951 \\
\hline Cholesterol & -0.019 & 0.881 & $1.238(0.655-2.339)$ & 0.213 & 0.511 \\
\hline Triglycerides & -0.183 & 0.144 & $1.831(0.841-3.948)$ & 0.605 & 0.127 \\
\hline HDL & 0.151 & 0.229 & $0.423(0.080-2.229)$ & -0.862 & 0.310 \\
\hline LDL & 0.007 & 0.955 & $1.157(0.560-2.390)$ & 0.145 & 0.694 \\
\hline AHI & -0.327 & 0.002 & $1.037(1.000-1.075)$ & 0.036 & 0.049 \\
\hline ODI & -0.371 & 0.002 & $1.042(1.001-1.085)$ & 0.041 & 0.044 \\
\hline TST90 & -0.251 & 0.044 & $1.126(0.966-1.311)$ & 0.118 & 0.128 \\
\hline Minimal $\mathrm{O}_{2}$ saturation & 0.282 & 0.023 & $0.921(0.850-0.998)$ & -0.082 & 0.045 \\
\hline Arousal index & -0.116 & 0.363 & $1.005(0.961-1.052)$ & 0.005 & 0.822 \\
\hline
\end{tabular}




\begin{tabular}{|c|c|c|c|}
\hline Variable & $\mathrm{B}(95 \% \mathrm{Cl})$ & Standardised $\boldsymbol{\beta}$ & $p$ value \\
\hline \multicolumn{4}{|c|}{ Stepwise linear regression } \\
\hline ODI & $-0.136(-0.182--0.09)$ & -0.351 & 0.005 \\
\hline Smoking pack-years & $-0.216(-0.131--0.301)$ & -0.305 & 0.014 \\
\hline \multicolumn{4}{|c|}{ Stepwise logistic regression } \\
\hline ODI & $1.047(1.001-1.095)$ & 0.046 & 0.045 \\
\hline
\end{tabular}

angiotensin-aldosterone system (RAAS) activity, oxidative stress, endothelial function and systemic inflammation. Only urinary norepinephrine as a marker of sympathetic activity correlated negatively with nocturnal systolic BP, and there was a trend towards significant correlation with the percentage of nocturnal dip ( $\mathrm{p}=0.087)$ and non-dipping BP status $(\mathrm{p}=0.058)$ (table 5).

\section{Effect of CPAP therapy on BP dip}

Thirty-one $(n=31)$ in the cohort were commenced on CPAP therapy, of which $20(n=20)$ agreed to return for repeat ABPM and assessment. The mean time on CPAP therapy at the return assessment was $18 \pm 6$ months. There was no change in BMI, no other diseases were diagnosed or medications introduced. Objective CPAP compliance data were available in 19 of 20 subjects (95\%), demonstrating a mean 90-day usage of $90.1 \pm 18.9 \%$ of nights. Mean usage hours per night over 90 days was $6.39 \pm 1.14$ hours. Average CPAP pressure was $10.7 \pm 1.5 \mathrm{cmH}_{2} \mathrm{O}$. CPAP therapy resulted in significant improvement in nocturnal systolic BP and BP dip (figure 1a, b). Furthermore, the number of subjects with a dipping pattern increased from 5 (25\%) to $9(45 \%)(p=0.109)$.

\section{Discussion}

The principal finding of this study is that OSA correlates with loss of nocturnal BP dipping and other BP variables in this cohort of patients who had no comorbidity and were normotensive based on 24-hour ABPM measurements. The nocturnal dip in BP as well as the per cent of non-dippers was more pronounced among the highest severity quartile of OSA and correlated strongly with the AHI and ODI. As a secondary end point, the data suggest that effective therapy with CPAP in this normotensive cohort may be associated with significant recovery of the nocturnal BP dipping pattern in addition to a lowering of systolic BP levels.

Our findings agree with previous reports that a non-dipping nocturnal blood pressure (BP) profile is particularly likely in patients with OSA [5], even in the absence of significant hypertension [8]. The

TABLE 5 Univariate correlation of \% blood pressure dip, nocturnal systolic blood pressure and non-dipping status with biomarkers of potential contributory pathways

\begin{tabular}{|c|c|c|c|c|c|c|c|}
\hline \multirow[b]{2}{*}{ Variable } & \multicolumn{2}{|c|}{$\%$ Blood pressure dip } & \multicolumn{2}{|c|}{$\begin{array}{l}\text { Nocturnal systolic blood } \\
\text { pressure }\end{array}$} & \multicolumn{3}{|c|}{ Non-dipping status } \\
\hline & $\begin{array}{l}\text { Correlation } \\
\text { coefficient }\end{array}$ & p-value & Correlation coefficient & $\mathrm{p}$-value & $\begin{array}{c}\text { Unstandardised } \boldsymbol{\beta} \\
(95 \% \mathrm{Cl})\end{array}$ & Standardised $\boldsymbol{\beta}$ & $p$ value \\
\hline Urinary norepinephrine & -0.242 & 0.087 & 0.387 & 0.007 & $1.006(1.000-1.013)$ & 0.006 & 0.058 \\
\hline Angiotensin II & 0.078 & 0.539 & -0.082 & 0.533 & $0.045(0.00-1688.09)$ & -3.096 & 0.564 \\
\hline Renin & 0.077 & 0.545 & 0.052 & 0.691 & $1.000(0.999-1.000)$ & 0.000 & 0.398 \\
\hline RHI & -0.100 & 0.482 & -0.004 & 0.981 & $1.277(0.425-3.544)$ & 0.204 & 0.706 \\
\hline Endothelin 1 & 0.099 & 0.438 & 0.025 & 0.849 & $0.775(0.584-1.027)$ & -0.255 & 0.076 \\
\hline CRP & -0.213 & 0.092 & 0.156 & 0.235 & $1.044(0.898-1.214)$ & 0.043 & 0.577 \\
\hline IL-6 & 0.022 & 0.864 & 0.064 & 0.626 & $1.115(0.906-1.374)$ & 0.109 & 0.304 \\
\hline TNF- $\boldsymbol{\alpha}$ & 0.147 & 0.248 & -0.058 & 0.661 & $1.003(0.939-1.072)$ & 0.003 & 0.923 \\
\hline
\end{tabular}



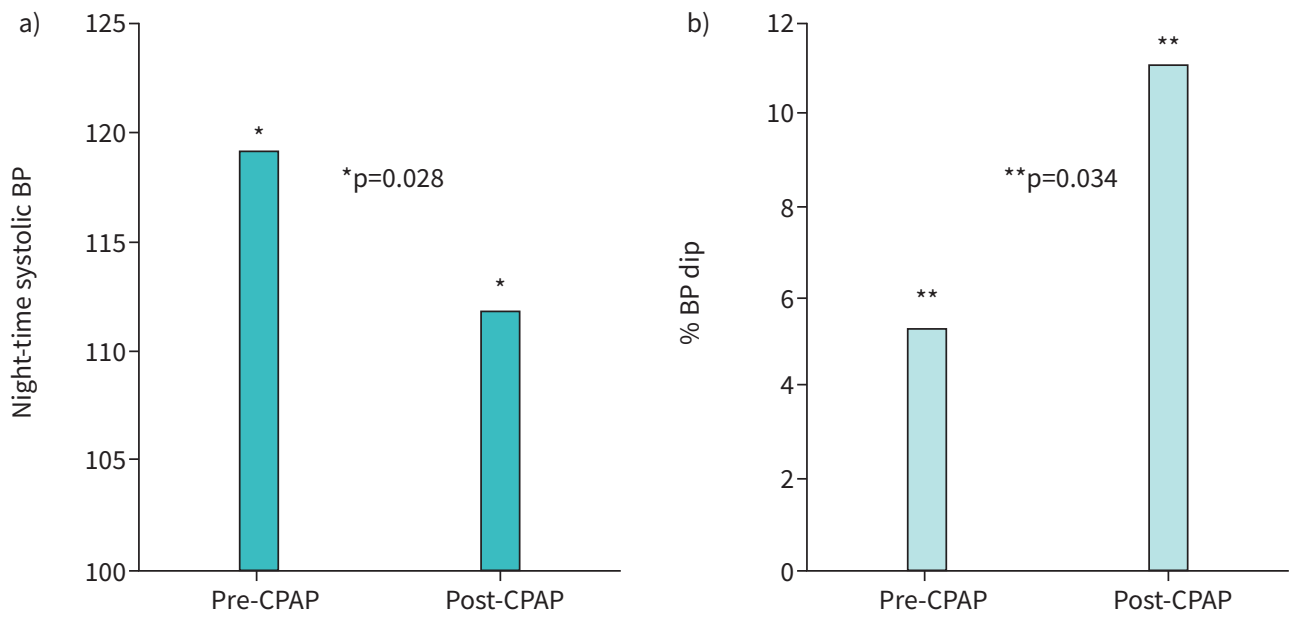

FIGURE 1 a) Night-time systolic blood pressure (BP) and b) per cent (\%) of BP dip in a subset of subjects preand post-treatment with continuous positive airways pressure (CPAP).

prevalence of a non-dipping BP was higher in our cohort than that reported in other studies $[5,7]$. However, these latter cohorts included treated hypertensive subjects and thus represent more heterogeneous populations. Data from the Wisconsin Sleep Cohort Study indicate a dose-response increase in the development of non-dipping hypertension with severity of OSA at baseline when followed for 7 years [7], which is confirmed by the report of SEIF and co-authors [5] demonstrating that in patients with established cardiovascular disease and moderate or severe OSA there is a $4 \%$ increase in the odds of having a non-dipping BP profile per unit increase in AHI. Furthermore, recent evidence from this department indicates that non-dipping nocturnal BP in hypertensive patients is a strong predictor of OSA, regardless of symptom profile [12].

The present data indicate that ODI was also an independent predictor of BP dip, consistent with previous research suggesting that measurements of oxygen desaturation, as a marker of OSA severity, are strongly associated with hypertension [13-15]. Furthermore, stepwise linear regression analysis showed that ODI was superior to AHI in predicting nocturnal blood pressure dip (table 4), which supports the previous report of TKacova and co-authors [14] that ODI is superior to AHI in the prediction of prevalent hypertension in a large sleep clinic population. Since ODI is the most relevant clinical measure of intermittent hypoxia, this finding supports intermittent hypoxia as a key OSA-related variable that predicts loss of nocturnal dipping in OSA.

Effective therapy of OSA with CPAP has been demonstrated to lower blood pressure [4], especially at night [16], although the overall reduction in BP with CPAP therapy is relatively small [17-20]. 24-hour ambulatory blood pressure monitoring has been reported to be a useful tool in identifying diurnal BP patterns to help predict and quantify BP response to CPAP therapy in OSA [21]. Although CPAP has been demonstrated to reduce the frequency of pre-hypertension or masked hypertension in patients with severe OSA [22], the ability of CPAP therapy in OSA patients to limit the future development of hypertension is not proven [23]. The potential ability of CPAP therapy to reverse a non-dipping BP in normotensive OSA and prevent future hypertension or cardiovascular events presents a possibility of primary prevention in this at-risk population.

Although only a secondary study end point, the present report demonstrating beneficial effects of CPAP on the nocturnal dipping BP profile in these normotensive OSA patients has implications for the future likelihood of cardiovascular morbidity in such patients. SAPIÑA-BELTRÁN and co-authors [9] identified patients with normal office BP measurements and demonstrated a reduction in BP in after 6 months of CPAP therapy. In this latter report, the benefits of CPAP therapy to BP variables principally related to nocturnal non-dippers and those with masked hypertension. Our study differed from this latter report in that no patient had masked hypertension, as all subjects had normal 24-hour BP levels prior to enrolment. Another randomised study of CPAP therapy in severe OSA patients with pre-hypertension demonstrated a reduction in office systolic and diastolic BP in treated patients compared to controls [22], and a recent meta-analysis confirms that elevated pre-CPAP BP values and age $<60$ years are factors more likely to 
predict a BP response in OSA [17]. Male gender, Epworth sleepiness scale and BMI have been identified as predictors of BP reduction following CPAP therapy in normotensive OSA [24]. Overall, our findings provide supportive evidence that CPAP therapy is beneficial in the primary prevention of hypertension in patients with OSA and are especially relevant in the context of the recent SAVE trial [10], which reported that CPAP was ineffective in the secondary prevention of cardiovascular morbidity and mortality among patients with established cardiovascular disease. Further controlled prospective studies of CPAP therapy in normotensive OSA populations will be required to confirm this potential benefit.

Potential intermediate mechanisms involved in the development of hypertension in OSA are likely multi-factorial, but sympathetic activation appears particularly important [4]. Other proposed mechanisms include activation of the renin-angiotensin-aldosterone system (RAAS) [25], systemic and vascular inflammation, oxidative stress and endothelial dysfunction [26], although specific evidence relating these intermediate mechanisms to non-dipping nocturnal BP is limited [27]. We evaluated a range of potential intermediate mechanisms in our study population, but the only significant finding was a significant relationship between overnight urinary norepinephrine, which is a marker of sympathetic excitation, and nocturnal systolic BP levels. However, urinary norepinephrine showed only a non-significant trend towards a relationship with nocturnal BP dipping status. These findings agree with several previous reports indicating elevated urinary epinephrine levels and other measures of sympathetic excitation in OSA [28, 29]. No other significant relationship was found across the range of variables studied in our patient population, and thus our data provide no further insight into the mechanisms of BP dipping in these subjects.

The present report has several important strengths. The study cohort represents a carefully selected consecutive group of patients referred for investigation of suspected OSA who were free of all other comorbidities and were not taking any regular medication. All patients were normotensive based on 24-hour mean systolic and diastolic BP levels. These findings together with those of previous reports [9] suggest that large patient numbers may not be necessary to identify potential benefit of CPAP therapy for OSA in the primary prevention of hypertension.

The study also has several limitations. First, only male subjects were studied and thus the findings cannot be extrapolated to female OSA patients. This aspect is relevant in the context of recent reports indicating sex differences in clinical OSA phenotypes [30] and in predisposition to incident hypertension [31]. Second, not all patients who were commenced on CPAP therapy for OSA returned for follow-up. This failure was despite repeated efforts by the lead investigator to contact these patients, some of whom could not be contacted, and others declined to repeat the initial protocol after a period on CPAP therapy. Thus, we cannot exclude the possibility of selection bias in those patients who returned for follow-up evaluation. Thirdly, the variables collected to represent intermediate mechanisms in the development of OSA were not repeated in the cohort of patients treated with CPAP, so no conclusions can be made as to whether CPAP affected these variables.

In conclusion, this report confirms the strong association in a normotensive cohort between OSA and nocturnal BP and supports intermittent hypoxia as an important mechanistic pathway. These findings also provide supportive evidence that CPAP therapy may play a significant role in the primary prevention of hypertension in OSA patients, although further studies are required to confirm this possibility.

Submitted article, peer reviewed.

Conflict of interest: None declared.

Support statement: This study was supported by Health Research Board grant HPF-2012-17. Funding information for this article has been deposited with the Crossref Funder Registry.

\section{References}

1 Benjafield AV, Ayas NT, Eastwood PR, et al. Estimation of the global prevalence and burden of obstructive sleep apnoea: a literature-based analysis. Lancet Respir Med 2019; 7: 687-698.

2 Cutler JA, Sorlie PD, Wolz M, et al. Trends in hypertension prevalence, awareness, treatment, and control rates in United States adults between 1988-1994 and 1999-2004. Hypertension 2008; 52: 818-827.

3 Thomas SJ, Booth JN, 3rd, Jaeger BC, et al. Association of sleep characteristics with nocturnal hypertension and nondipping blood pressure in the CARDIA study. J Am Heart Assoc 2020; 9: e015062. 
4 Parati G, Lombardi C, Hedner J, et al. Recommendations for the management of patients with obstructive sleep apnoea and hypertension. Eur Respir J 2013; 41: 523-538.

5 Seif F, Patel SR, Walia HK, et al. Obstructive sleep apnea and diurnal nondipping hemodynamic indices in patients at increased cardiovascular risk. J Hypertens 2014; 32: 267-275.

6 Wolf J, Hering D, Narkiewicz K. Non-dipping pattern of hypertension and obstructive sleep apnea syndrome. Hypertens Res 2010; 33: 867-871.

7 Hla KM, Young T, Finn L, et al. Longitudinal association of sleep-disordered breathing and nondipping of nocturnal blood pressure in the Wisconsin Sleep Cohort Study. Sleep 2008; 31: 795-800.

8 Sasaki N, Ozono R, Edahiro Y, et al. Impact of non-dipping on cardiovascular outcomes in patients with obstructive sleep apnea syndrome. Clin Exp Hypertens 2015; 37: 449-453.

9 Sapiña-Beltrán E, Santamaria-Martos F, Benítez I, et al. Normotensive patients with obstructive sleep apnoea: changes in 24-h ambulatory blood pressure monitoring with continuous positive airway pressure treatment. J Hypertens 2019; 37: 720-727.

10 McEvoy RD, Antic NA, Heeley E, et al. CPAP for prevention of cardiovascular events in obstructive sleep apnea. N Engl J Med 2016; 375: 919-931.

11 Berry RB, Budhiraja R, Gottlieb DJ, et al. Rules for scoring respiratory events in sleep: update of the 2007 AASM Manual for the Scoring of Sleep and Associated Events. Deliberations of the Sleep Apnea Definitions Task Force of the American Academy of Sleep Medicine. J Clin Sleep Med 2012; 8: 597-619.

12 Crinion SJ, Ryan S, Kleinerova J, et al. Nondipping nocturnal blood pressure predicts sleep apnea in patients with hypertension. J Clin Sleep Med 2019; 15: 957-963.

13 Wang N, Meng Z, Ding N, et al. Oxygen desaturation rate as a novel intermittent hypoxemia parameter in severe obstructive sleep apnea is strongly associated with hypertension. J Clin Sleep Med 2020; 16: 1055-1062.

14 Tkacova R, McNicholas WT, Javorsky M, et al. Nocturnal intermittent hypoxia predicts prevalent hypertension in the European Sleep Apnoea Database cohort study. Eur Respir J 2014; 44: 931-941.

15 Natsios G, Pastaka C, Vavougios G, et al. Age, body mass index, and daytime and nocturnal hypoxia as predictors of hypertension in patients with obstructive sleep Apnea. J Clin Hypertens (Greenwich) 2016; 18: 146-152.

16 Joyeux-Faure M, Baguet JP, Barone-Rochette G, et al. Continuous positive airway pressure reduces night-time blood pressure and heart rate in patients with obstructive sleep apnea and resistant hypertension: the RHOOSAS randomized controlled trial. Front Neurol 2018; 9: 11.

17 Pengo MF, Soranna D, Giontella A, et al. Obstructive sleep apnoea treatment and blood pressure: which phenotypes predict a response? A systematic review and meta-analysis. Eur Respir J 2020; 55: 1901945.

$18 \mathrm{Hu} \mathrm{XY}$, Fan JQ, Chen SJ, et al. The role of continuous positive airway pressure in blood pressure control for patients with obstructive sleep apnea and hypertension: a meta-analysis of randomized controlled trials. J Clin Hypertens 2015; 17: 215-222.

19 Duran-Cantolla J, Aizpuru F, Montserrat JM, et al. Continuous positive airway pressure as treatment for systemic hypertension in people with obstructive sleep apnoea: randomised controlled trial. Br Med J 2010; 341: 9.

20 Schein ASO, Kerkhoff AC, Coronel CC, et al. Continuous positive airway pressure reduces blood pressure in patients with obstructive sleep apnea: a systematic review and meta-analysis with 1000 patients. J Hypertens 2014; 32: 1762-1773.

21 Castro-Grattoni AL, Torres G, Martínez-Alonso M, et al. Blood pressure response to CPAP treatment in subjects with obstructive sleep apnoea: the predictive value of 24-h ambulatory blood pressure monitoring. Eur Respir J 2017; 50: 1700651.

22 Drager LF, Pedrosa RP, Diniz PM, et al. The effects of continuous positive airway pressure on prehypertension and masked hypertension in men with severe obstructive sleep apnea. Hypertension 2011; 57: 549-545.

23 Barbe F, Duran-Cantolla J, Sanchez-de-la-Torre M, et al. Effect of continuous positive airway pressure on the incidence of hypertension and cardiovascular events in nonsleepy patients with obstructive sleep apnea: a randomized controlled trial. JAMA 2012; 307: 2161-2168.

24 Yorgun $\mathrm{H}$, Kabakçı G, Canpolat U, et al. Predictors of blood pressure reduction with nocturnal continuous positive airway pressure therapy in patients with obstructive sleep apnea and prehypertension. Angiology 2014; 65: 98-103.

25 Jin ZN, Wei YX. Meta-analysis of effects of obstructive sleep apnea on the renin-angiotensin-aldosterone system. J Geriatr Cardiol 2016; 13: 333-343.

26 McNicholas WT, Bonsignore MR. Sleep apnoea as an independent risk factor for cardiovascular disease: current evidence, basic mechanisms and research priorities. Eur Respir J 2007; 29: 156-178.

27 Crinion SJ, Ryan S, McNicholas WT. Obstructive sleep apnoea as a cause of nocturnal nondipping blood pressure: recent evidence regarding clinical importance and underlying mechanisms. Eur Respir J 2017; 49: 1601818.

28 Carlson JT, Hedner J, Elam M, et al. Augmented resting sympathetic activity in awake patients with obstructive sleep apnea. Chest 1993; 103: 1763-1768. 
29 Narkiewicz K, Somers VK. The sympathetic nervous system and obstructive sleep apnea: implications for hypertension. J Hypertens 1997; 15: 1613-1619.

30 Basoglu OK, Tasbakan MS. Gender differences in clinical and polysomnographic features of obstructive sleep apnea: a clinical study of 2827 patients. Sleep Breath 2018; 22: 241-249.

31 Cano-Pumarega I, Barbé F, Esteban A, et al. Sleep apnea and hypertension: are there sex differences? The Vitoria sleep cohort. Chest 2017; 152: 742-750. 